\title{
Calcitonin gene-related peptide receptor antagonist BIBN4096BS regulates synaptic transmission in the vestibular nucleus and improves vestibular function via PKC/ ERK/CREB pathway in an experimental chronic migraine rat model
}

Ruimin Tian'1, Yun Zhang ${ }^{1}$, Qi Pan'1, Yunfeng Wang 1,2, Qianwen Wen ${ }^{3}$, Xiaoping Fan'1, Guangcheng Qin³, Dunke Zhang ${ }^{3}$, Lixue Chen ${ }^{3}$, Yixin Zhang ${ }^{1 *}$ (D) and Jiying Zhou'

\begin{abstract}
Background: Vestibular symptoms are frequently reported in patients with chronic migraine (CM). However, whether vestibular symptoms arise through overlapping neurobiology of migraine remains to be elucidated. The neuropeptide calcitonin gene-related peptide (CGRP) and CGRP1 receptor play important pathological roles in facilitating central sensitization in CM. Therefore, we aimed to investigate whether CGRP1 receptor contributes to vestibular dysfunction after CM by improving synaptic transmission in the vestibular nucleus (VN).

Methods: A CM rat model was established by recurrent intermittent administration of nitroglycerin (NTG). Migraineand vestibular-related behaviors were assessed. CGRP1 receptor specific antagonist, BIBN4096BS, and protein kinase C (PKC) inhibitor chelerythrine chloride (CHE) were administered intracerebroventricularly. The expressions of CGRP and CGRP1 receptor components, calcitonin receptor-like receptor (CLR) and receptor activity modifying protein 1 (RAMP1) were evaluated by western blot, immunofluorescent staining and quantitative real-time polymerase chain reaction in the vestibular nucleus (VN). Synaptic associated proteins and synaptic morphological characteristics were explored by western blot, transmission electron microscope, and Golgi-cox staining. The expressions of PKC, phosphorylated extracellular signal regulated kinase ( $\mathrm{p}$-ERK), phosphorylated CAMP response element-binding protein at serine 133 site (p-CREB-S133) and c-Fos were detected using western blot or immunofluorescent staining.

Results: The expressions of CGRP, CLR and RAMP1 were significantly upregulated in CM rats. CLR and RAMP1 were expressed mainly in neurons. BIBN4096BS treatment and PKC inhibition alleviated mechanical allodynia, thermal hyperalgesia and vestibular dysfunction in CM rats. Additionally, BIBN4096BS treatment and PKC inhibition markedly inhibited the overexpression of synaptic associated proteins and restored the abnormal synaptic structure in VN after
\end{abstract}

\footnotetext{
*Correspondence: zhangyx_cq@163.com

${ }^{1}$ Department of Neurology, The First Affiliated Hospital of Chongqing

Medical University, 1st You Yi Road, Yu Zhong District, 400016 Chongqing,

China

Full list of author information is available at the end of the article
} permits use, sharing, adaptation, distribution and reproduction in any medium or format, as long as you give appropriate credit to the original author(s) and the source, provide a link to the Creative Commons licence, and indicate if changes were made. The images or other third party material in this article are included in the article's Creative Commons licence, unless indicated otherwise in a credit line to the material. If material is not included in the article's Creative Commons licence and your intended use is not permitted by statutory regulation or exceeds the permitted use, you will need to obtain permission directly from the copyright holder. To view a copy of this licence, visit http://creativecommons.org/licenses/by/4.0/. The Creative Commons Public Domain Dedication waiver (http://creativeco mmons.org/publicdomain/zero/1.0/) applies to the data made available in this article, unless otherwise stated in a credit line to the data. 
CM. Furthermore, BIBN4096BS treatment dysregulated the expression levels of PKC, p-ERK and p-CREB-S133, and attenuated neuronal activation in VN after CM.

Conclusions: The present study demonstrated that CGRP1 receptor inhibition improved vestibular function after CM by reversing the aberrant synaptic transmission via downregulating PKC/ERK/CREB signaling pathway. Therapeutic interventions by inhibiting CGRP/CGRP1 signaling may be a new target for the treatment of vestibular symptoms in CM.

Keywords: CGRP, Migraine, Vestibular dysfunction, Vestibular nucleus, Central sensitization, Synaptic transmission

\section{Introduction}

Patients with migraine usually experience not only headache but also balance problems and dizziness, i.e. motion sickness and recurrent vertigo $[1,2]$. With headache frequency increased, the incidence of dizziness and associated handicap significantly elevated [2]. Of note, up to $85 \%$ of patients with chronic migraine $(\mathrm{CM})$ reported vestibular symptoms [2]. Unfortunately, most migraine patients with vestibular symptoms do not respond well to treatment with traditional vestibular suppressants [3]. Despite this, it is not yet understood whether vestibular symptoms arise through overlapping neurobiology or independent mechanisms of migraine. Consequently, the application of migraine-specific treatments remains limited in this population [4]. Central sensitization of trigeminovascular neurons in the trigeminal nucleus caudalis (TNC) is a leading culprit that contributes to sensory hypersensitivity in migraine [5], while the enhancement of synaptic transmission is the neural basis of central sensitization in rat models of CM $[6,7]$. Our previous study demonstrated that central sensitization in the vestibular nucleus $(\mathrm{VN})$ was the potential mechanism for vestibular symptoms in migraine, and vestibular dysfunction was significantly related to neuronal activation in $\mathrm{VN}$ [8]. Thus, effectively suppressing neuronal activation via modulating synaptic transmission efficiency in $\mathrm{VN}$ might be a promising approach in the treatment of vestibular symptoms in migraine patients.

The neuropeptide calcitonin gene-related peptide (CGRP) has been demonstrated to play an essential role in facilitating pain transmission [9]. CGRP is a 37-amino acid neuropeptide, and produced mainly by neurons [10]. CGRP mediates its function primarily by activating CGRP1 receptor, containing calcitonin receptor-like receptor (CLR), a G protein-coupled receptor (GPCR), together with receptor activity modifying protein 1 (RAMP1), a single transmembrane protein which is required for functional expression of CLR at the cell surface $[11,12]$. Prior study found that the expression level of CGRP in VN increased significantly in a rat model of motion sickness, whilst anisodamine could markedly suppress the CGRP expression in VN and reverse motion sickness-related behaviors [13].
Our previous study showed similar results in CM rats, and blunting CGRP synthesis in VN caused a marked reversal of nitroglycerin (NTG)-induced vestibular dysfunction [8]. To date, clinical trials targeting CGRP and its receptors witness a great success in migraine treatment [14]. However, whether CGRP and its receptors are viable therapeutic targets in migraine patients with vestibular symptoms is uncertain, and whether CGRP antibodies and CGRP1 receptor antagonists can alleviate vestibular symptoms in migraine patients requires further investigation.

CGRP has been demonstrated to produce long-term potentiation (LTP) in the insular cortex and anterior cingulate cortex [15]. This phenomenon could be significantly blocked by specific CGRP1 receptor complex antagonists, $\mathrm{CGRP}_{8-37}$ or BIBN4096BS, which further achieving analgesic effects $[15,16]$. Besides that, these two antagonists could also reverse pain-related synaptic function in the amygdala of arthritic rats, as evidenced by the reduced neuronal excitability and decreased amplitude of miniature excitatory postsynaptic currents (mEPSCs) [17], pointing that CGRP and CGRP1 receptor signaling might modulate synaptic function in CM. Although CGRP1 receptor is generally considered a $\mathrm{G \alpha}_{\mathrm{s}}-$ coupled GPCR, it is becoming clear that it also couples to additional $G$ proteins and other proteins [12, 18]. In vitro studies showed that CGRP activated protein kinase $C$ (PKC) and extracellular signal regulated kinase (ERK) in HEK293 cells, and pre-incubation spinal cord slices with specific ERK or PKC inhibitor significantly shortened CGRP-stimulated firing time of lamina I neurons [19]. Moreover, cAMP response element-binding protein (CREB), one of the major downstream mediators of ERK, has been considered as an important indicator of central sensitization in CM rats [20,21]. Downregulation of phosphorylated CREB at Serine 133 site (p-CREB-S133) was significantly associated with the reduction of neuronal activation in TNC after CM [21]. These data suggests that CGRP and CGRP1 receptor modulate synaptic transmission in CM rats might through PKC/ERK/CREB signaling pathway.

Based on the above-mentioned evidence, we hypothesized that CGRP1 receptor antagonist BIBN4096BS 
would reduce neuronal activation in $\mathrm{VN}$ and alleviate vestibular dysfunction in rat models of $\mathrm{CM}$ and that these beneficial effects might be mediated by PKC/ERK/ CREB signaling. Through these attempts, we explore the therapeutic efficacy of CGRP1 receptor antagonist in a $\mathrm{CM}$ model as a preclinical approach for potential clinical translation in migraine patients with vestibular symptoms.

\section{Materials and methods}

\section{Animals}

All experiments were approved by the Commission of Chongqing Medical University for ethics of experiments on animals and followed the National Institutes of Health Guide for the Care and Use of Laboratory Animals. All animal experiments complied with the ARRIVE guidelines. One-hundred and eight male Sprague Dawley (SD) rats weighing 250-300 g were obtained from Hunan Slack Jingda Experimental Animal Co., Ltd. (Hunan, China). They were kept in the standard condition of $12 \mathrm{~h}$ light-dark cycle with the temperature at $23^{\circ} \mathrm{C} \pm 2{ }^{\circ} \mathrm{C}$ and $50 \pm 5 \%$ relative humidity of the environment. Water and food were adequately provided. The rats were randomly assigned to each group after a week of acclimatization.

\section{Establishment of the chronic migraine model}

The general procedure for inducing CM in male SD rats via nitroglycerin (NTG) injection was performed as previously described [8]. In brief, $5 \mathrm{mg} / \mathrm{ml}$ NTG (Beijing Regent, China) that was dissolved in 30\% alcohol, 30\% propylene glycol and water was prepared as stock solution. Prior to each injection, NTG stock solution was diluted to $1 \mathrm{mg} / \mathrm{ml}$ with $0.9 \%$ saline for injection use. All rats were randomly received intraperitoneal injection of $10 \mathrm{mg} / \mathrm{kg}$ of NTG or $0.9 \%$ saline at an equal volume every other day for 9 days (five times in total). According to the previous study, the mechanical thresholds were comparable between rats that received $0.9 \%$ saline and $6 \%$ propylene glycol $+6 \%$ alcohol $+0.9 \%$ saline [22]. After the fifth injection, rats were then placed back to the cages until sacrifice.

\section{Drug administration}

BIBN4096BS (MedChemExpress, HY-10,095 A), a specific CGRP1 receptor antagonist, and chelerythrine chloride (CHE) (MedChemExpress, HY-12,048), a PKC inhibitor, were injected into the left lateral ventricle by intracerebroventricular administration as described previously [23]. BIBN4096BS was dissolved in 10\% DMSO, $40 \%$ PEG300, 5\% Tween 80 and $45 \%$ saline to a final concentration of $0.01 \mu \mathrm{g} / \mu \mathrm{l}$, which was administered at a dose of $0.1 \mu \mathrm{g} /$ day/rat $2 \mathrm{~h}$ before each NTG injection based on previous study [24]. The CHE was dissolved in the same solution as BIBN4096BS to a final concentration of $1 \mathrm{mmol} / \mathrm{L}$, which was administered at a dose of $10 \mathrm{nmol} /$ day/rat based on previous study [25]. And the $\mathrm{CHE}$ was intracerebroventricularly injected same as the BIBN4096BS administration route, that is, injected $2 \mathrm{~h}$ prior each NTG administration. An equivalent volume of solvent $(10 \mu \mathrm{l})$ was administered intracerebroventricularly as a corresponding vehicle control group $(\mathrm{CM}+$ vehicle).

\section{Stereotaxic surgery procedures and intracerebroventricular injection}

As described previously, rats were anesthetized with $10 \%$ chloral hydrate $(4 \mathrm{ml} / \mathrm{kg}$, intraperitoneal) combined with analgesics $(0.01 \mathrm{mg} / \mathrm{kg}$ buprenorphine) [8]. When the pedal reflex was absent, they were fixed in a stereotactic frame (ST-51,603; Stoelting Co, Chicago, IL, USA) with a heating pad. The skin was incised on the longitudinal plane to expose the bregma. A cranial burr hole with a diameter of $1 \mathrm{~mm}$ was performed above the left dura at the following coordinates aiming for the left lateral ventricle: $1.0 \mathrm{~mm}$ rear from the bregma and $1.5 \mathrm{~mm}$ left lateral to the bregma. A sterile cannula with a screw cap was implanted at the above coordinates and stabilized with dental cement [21]. The animals were allowed to recover for at least 7 days until their thermal and mechanical pain thresholds returned to the preoperative levels.

Intracerebroventricular injections were performed in anesthetized rats using a $10-\mu \mathrm{l}$ Hamilton syringe through the cannula at following stereotaxic coordinates: $1.0 \mathrm{~mm}$ rear from the bregma, $1.5 \mathrm{~mm}$ left lateral to the bregma, and $4.0 \mathrm{~mm}$ from the skull plane. Before and after each injection, the patency of the guide cannula was checked. The infusion was performed at a rate of $2 \mu \mathrm{l} / \mathrm{min}$. After infusion, the needle was left in place for additional $10 \mathrm{~min}$ and then was withdrawn over $1 \mathrm{~min}$. Next, animals were placed back to the heating pads for recovery and subsequent NTG injection.

\section{Experimental design and animal groups Experiment 1}

To evaluate endogenous expression levels of CGRP and CGRP1 receptor components (CLR and RAMP1), rats were randomly divided into two groups to perform western blot or quantitative real-time polymerase chain reaction (qPCR) and immunofluorescence staining: Saline and $C M(n=6 /$ group). Rats were subjected to behavioral tests $15-20$ min prior and $2 \mathrm{~h}$ post NTG injection. And the basal vestibular function test was performed on the day before NTG administration. In addition, the cellular co-localizations of CLR and RAMP1 were evaluated using double immunofluorescence staining in Saline and CM group ( $\mathrm{n}=3$ /group). 


\section{Experiment 2}

The neuroprotective effects of CGRP1 receptor antagonist treatment (BIBN4096BS) for CM were evaluated. Rats were randomly divided into four groups: Saline, $\mathrm{CM}, \mathrm{CM}+$ vehicle and CM+BIBN4096BS ( $\mathrm{n}=6$ /group). Behavioral tests were performed 15-20 min prior intracerebroventricular injection and $2 \mathrm{~h}$ post NTG injection, and then sacrificed to collect $\mathrm{VN}$ tissues for western blot, qPCR or immunofluorescence staining to evaluate the expression levels of CGRP, CLR, RAMP1 and synaptic associated proteins (post-synaptic density protein 95: PSD95; synaptophysin: Syp; synaptotagmin-1: Syt-1), for transmission electron microscope scanning to evaluate the thickness of postsynaptic density (PSD), length of synaptic activity zone and synaptic interface curvature, and for Golgi-cox staining to evaluate dendritic spines density in VN.

\section{Experiment 3}

To assess the potential molecular pathway of CGRP1 receptor in anti-neuronal activation effect, the specific PKC inhibitor, $\mathrm{CHE}$, was administered to CM rats. The groups included Saline, CM, CM+vehicle, $\mathrm{CM}+\mathrm{BIBN4096BS}$ and $\mathrm{CM}+\mathrm{CHE}$ ( $\mathrm{n}=6$ /group). The administration route of BIBN4096BS and CHE was same as that in Experiment 2. Behavioral tests were performed 15-20 min prior intracerebroventricular injection and $2 \mathrm{~h}$ post NTG administration. The VN tissues were collected for western blot to measure protein levels of PKC, phosphorylated ERK, phosphorylated CREB-S133 and synaptic associated proteins (PSD95, Syp and Syt-1), and for immunofluorescence staining to detect neuronal activation.

\section{Behavioral tests}

All behavioral studies were performed by two investigators who were blinded to the treatment groups as described previously [8]. Basal responses of mechanical and thermal stimuli were performed 15-20 min prior to each NTG administration or intracerebroventricular injection. Post-treatment responses of mechanical allodynia and thermal hyperalgesia, balance beam walk and negative geotaxis were performed at $2 \mathrm{~h}$ after each NTG administration. Before starting to record the data of balance beam walk and negative geotaxis tests, the rats received three days of adaptive training, and the data of basal vestibular function test was recorded on the day before NTG administration.

\section{Assessment of mechanical allodynia}

Tactile sensitivity was measured using the electronic von Frey instrument (Electrovonfrey, 2391, IITC Inc.,
Woodland Hills, CA, USA) as previous described [21]. Briefly, the pressure probe tip was applied perpendicularly to periorbital region that was over the rostral portion of the eyes, as well as the central area of hind paws surface from a small force value and gradually increasing. The thresholds were automatically recorded when the rat's head or paw withdrawal was observed. After the initial positive response, repeated stimulations were performed as the same pattern. There was an interval of $5 \mathrm{~min}$ between applications. For each rat, three positive responses were obtained to calculate the average threshold.

\section{Assessment of thermal hyperalgesia}

Thermal sensitivity was assessed using Hargreaves radiant heat apparatus (model PL-200, IITC, Taimeng, Chengdu, China) as described previously [8]. Briefly, the rats were placed in a transparent cage to acclimate for $30 \mathrm{~min}$. Then, infrared radiation (intensity: 20\%), which caused an abrupt withdraw, was applied to the central part of hind paws. Withdrawal latency was automatically recorded by the device. The average of three records with an interval of $5 \mathrm{~min}$ was used to determine the withdrawal latency to the heat.

\section{Balance beam walk}

To evaluate the motor coordination and balance, balance beam walk was performed as previous described [26]. A balance beam of $190 \mathrm{~cm}$ in length and $2.5 \mathrm{~cm}$ in diameter was placed horizontally at $40 \mathrm{~cm}$ above the table. A cushion was placed below to protect the fallen animals. The duration that rats used to pass through the beam was recorded, and the maximum recording time was $15 \mathrm{~s}$ (three trials per rat with the interval of $90 \mathrm{~s}$ between each trial).

\section{Negative geotaxis}

Negative geotaxis, an automatic unlearned response and directional movement against gravitational cues, is stimulated by the abnormal position of the head and body, initiated by vestibular and postural systems [27, 28]. This test is considered as a diagnostic of vestibular function, reflex development, and motor skills [26]. During this experiment, we used a sponge pad with inclined surface and covered it with a rough towel. As described previously, animals were placed on a $40^{\circ}$ inclined surface (ranging from $15^{\circ}$ to $70^{\circ}$ in most tests) with their head downwards [8]. And the hind paws of rats were about $15 \mathrm{~cm}$ away from the top of the slope. The duration for a turn of $180^{\circ}$ upward was recorded. The maximum recording time was $15 \mathrm{~s}$ ( 3 trials per rat). And the average of three records with an interval of 1 min was used. 
Quantitative real-time polymerase chain reaction (qPCR) According to previous studies $[8,26]$, the $\mathrm{VN}$ tissues (localized between -10 and $-12 \mathrm{~mm}$ from bregma) were quickly extracted based on the rat brain atlas of Paxinos and Waston (6th edition) and stored in liquid nitrogen immediately for qPCR. In brief, total RNA from VN tissues was extracted via RNAiso Plus reagent (TaKaRa, Dalian, China) and quantified by the NanoDrop kit (Thermo, USA). Then, the cDNA synthesis was conducted using the PrimeScript ${ }^{\mathrm{TM}}$ RT Kit (TaKaRa, Dalian, China). Finally, the mRNA expression of CLR and RAMP1 were detected on a CFX96 Touch thermocycler (Bio-Rad, CA, USA) using SYBR Premix Ex Taq TM II (TaKaRa, Dalian, China). Relative mRNA levels were analyzed using the $\Delta \Delta \mathrm{Cq}$ method with GAPDH as an internal control. The primer sequences for CLR, RAMP1 and GAPDH (Sangon Biotech, Shanghai, China) were as follows: CLR: F: $5^{\prime}$-CTCATT GTGGTGGCTGTGTTTGC-3'， R: 5'-GCAGGCAGG AAGCAGAGGAAAC-3'; RAMP1: F: 5'-AGGGAA GACTCTGTGGTGTGACTG-3', R: 5'-GTAGCGGTG GTGGACAGCAATG-3'; GAPDH: F: 5'-ATGACTCTA CCCACGGCAAGCT-3', R: 5'-GGATGCAGGGAT GATGTTCT-3'.

\section{Western blot analysis}

As described previously [21], the $\mathrm{VN}$ tissues were homogenized in radioimmunoprecipitation assay (RIPA) lysis buffer (Beyotime, Shanghai, China) mixed with protease inhibitor phenylmethylsulfonyl fluoride (PMSF, Beyotime, Shanghai, China) for $1 \mathrm{~h}$ at $4{ }^{\circ} \mathrm{C}$, following centrifugation at $12,000 \times \mathrm{g}$ in $4{ }^{\circ} \mathrm{C}$ for $15 \mathrm{~min}$. The bicinchoninic acid (BCA) protein assay kit (Beyotime, Shanghai, China) was used to detect the protein concentration of supernatant. Equal amount of protein samples (30 $\mu \mathrm{g}$ per lane) was loaded on the SDS-PAGE gels (Beyotime, Shanghai, China) for electrophoresis about $2 \mathrm{~h}$, subsequent transferred to the PVDF membranes (Millipore, USA). These membranes were blocked for $2 \mathrm{~h}$ in Tris-buffered saline containing Tween 20 (TBST) containing 5\% non-fat milk at room temperature and then incubated with primary antibodies overnight at $4{ }^{\circ} \mathrm{C}$. The next day, these membranes were incubated with horseradish peroxidase-conjugated secondary antibodies (goat-anti-rabbit, goat-antimouse) diluted in TBST for $1 \mathrm{~h}$ at room temperature. The immunoreactive bands were visualized by BeyoECL Plus kit (Beyotime, Shanghai, China), and analyzed by an imaging system (Fusion, Germany). GAPDH and $\beta$-actin were used as the internal reference to normalize the relative expression levels of target proteins. The details for each antibody used for western blot are shown in Table 1.

\section{Immunofluorescence staining}

The tissues were collected $2 \mathrm{~h}$ after the final NTG injection to detect the expression of c-Fos, while for other targets, tissues were collected within $12 \mathrm{~h}$. After general anesthesia, the rats were perfused intracardially with $0.1 \mathrm{M}$ PBS $250 \mathrm{~mL}$, followed by $250 \mathrm{~mL}$ of $4 \%$ paraformaldehyde (PFA). According to previous study [8], the TNC (localized between -14 and $-16 \mathrm{~mm}$ from bregma according to the rat brain atlas of Paxinos and Waston (6th edition)) and $\mathrm{VN}$ regions were immediately harvested. The tissues were postfixed in $4 \%$ PFA at $4{ }^{\circ} \mathrm{C}$ for $24 \mathrm{~h}$, and transferred to dehydration in $20 \%$ and then $30 \%$ sucrose until tissues sank. The transverse sections of TNC and VN tissues were sectioned at $15 \mu \mathrm{m}$ thickness on cryostat (Leica, Japan). The slices were permeabilized with $0.3 \%$ Triton $\mathrm{X}-100$ for $10 \mathrm{~min}$ at $37^{\circ} \mathrm{C}$ and blocked with $10 \%$ of goat serum (Boster, Wuhan, China) at $37{ }^{\circ} \mathrm{C}$ for $30 \mathrm{~min}$. Then, the slices were incubated with primary antibodies overnight at $4{ }^{\circ} \mathrm{C}$. After rinsing 3 times for $15 \mathrm{~min}$ in PBS, the sections were incubated with the species-specific fluorophore-labeled secondary antibodies for $90 \mathrm{~min}$ at $37{ }^{\circ} \mathrm{C}$. Finally, the $4^{\prime}, 6$-diamidino-2-phenylinodole (DAPI) (Beyotime, Shanghai, China) was employed to counterstain the nuclei. Images were acquired with a fluorescence confocal microscope (LSM800, Zeiss, Germany) equipped with structured illumination (Zen). The details for primary and secondary antibodies used for immunofluorescence staining are shown in Table 1.

Morphological identifications of TNC and VN were determined as previously described [8]. C-Fos+, CLR+, and RAMP1 + cells, as well as CGRP + fibers were quantified on both sides for TNC and VN from selected serial transverse sections that were collected from the rostral to caudal part. To avoid counting the same cell more than once, each section was separated by at least $250 \mu \mathrm{m}$ [8]. The number of target cells was assessed with x200 magnification through the optical fractionator method, while the fluorescence signal intensity of CGRP + fibers was determined using Image-pro Plus 6.2 software (Bethesda, MD, USA) ( $n=6$ rats per group, 5 images per rat).

\section{Transmission electron microscopy}

After general anesthesia, rats were intracardially perfused with $2.5 \%$ glutaraldehyde as previous described [21]. The VN tissues were collected and fixed by $4 \%$ glutaraldehyde at $4^{\circ} \mathrm{C}$ overnight. Next, the VN tissues were cut into $1 \mathrm{~mm}^{3}$ pieces and rinsed with PBS for $30 \mathrm{~min}$. The samples were post-fixed with $1 \%$ osmium tetroxide for $2 \mathrm{~h}$, followed by dehydrating in a graded series of ethanol $(50 \%, 70 \%, 90 \%, 100 \%$ and $100 \%$, each concentration for $10 \mathrm{~min}$ ) and transferring to $100 \%$ propylene 
Table 1 Antibodies used in western blot and immunofluorescence staining analysis

\begin{tabular}{|c|c|c|c|}
\hline Antibody & Manufacturer & Host & Dilution \\
\hline \multicolumn{4}{|l|}{ For Wertern blot analysis } \\
\hline CGRP & Abcam, UK & Rabbit & $1: 3000$ \\
\hline PKC & Bioss, China & Rabbit & 1:1000 \\
\hline PSD95 & Cell signaling technology, USA & Mouse & 1:1000 \\
\hline Synaptophysin & Abcam, UK & Rabbit & $1: 20000$ \\
\hline Synaptotagmin-1 & Bioss, China & Rabbit & 1:1000 \\
\hline p-ERK & Cell signaling technology, USA & Rabbit & $1: 2000$ \\
\hline ERK & Cell signaling technology, USA & Rabbit & $1: 1000$ \\
\hline p-CREB-133 & Abcam, UK & Rabbit & $1: 5000$ \\
\hline CREB & Wanleibio, China & Rabbit & 1:1000 \\
\hline GAPDH & ZEN BIO, China & Mouse & $1: 8000$ \\
\hline$\beta$-actin & ZSGB BIO, China & Mouse & $1: 1500$ \\
\hline Anti-rabbit lgG (HRP) & ZEN BIO, China & Goat & $1: 5000$ \\
\hline Anti-mouse lgG (HRP) & ZEN BIO, China & Goat & $1: 5000$ \\
\hline \multicolumn{4}{|l|}{ For Immunofluorescent staining } \\
\hline CGRP & Santa Cruz, USA & Mouse & $1: 100$ \\
\hline CLR & Bioss, China & Rabbit & $1: 1000$ \\
\hline RAMP1 & Proteintech, China & Rabbit & $1: 200$ \\
\hline c-Fos & Novus Biologicals, USA & Rabbit & $1: 5000$ \\
\hline NeuN & Abcam, UK & Mouse & $1: 500$ \\
\hline GFAP & Santa Cruz, USA & Mouse & $1: 200$ \\
\hline Iba-1 & ThermoFisher, USA & Mouse & $1: 500$ \\
\hline Cy3-conjugatedgoat anti-rabbit lgG & Beyotime, China & Goat & $1: 500$ \\
\hline Alexa Fluor 488 goat anti-mouse lgG & Beyotime, China & Goat & $1: 500$ \\
\hline
\end{tabular}

oxide for $15 \mathrm{~min}$, and then embedded in Epon-Araldite resin (Canemco \& Marivac, Lakefield, Quebec, Canada). Ultrathin Sects. (80-90 nm) were prepared with an ultramicrotome (Reichert-Jung, Inc., Cambridge, UK). Then sections were washed twice with distilled water, and counterstained with $2 \%$ uranyl acetate containing with lead citrate for $45 \mathrm{~min}$. Sections were visualized on a transmission electron microscope (JEM-1400 PLUS, JEOL Ltd., Japan).

The thickness of the postsynaptic density (PSD) and length of synaptic activity zone, as well as the curvature of synaptic interface were measured in accordance with previous protocol [29]. The observer, who was blinded to the experiment groups, analyzed images using Image-pro Plus 6.2 software (Bethesda, MD, USA). Each group contained 3 rats, and 3 ultrathin sections were obtained from each rat. Five images per section were obtained to estimate the average values.

\section{Golgi-cox staining}

The morphological changes of neuronal dendritic spines were determined through Golgi-cox staining. An FD Rapid Golgi Stain $\mathrm{Kit}^{\mathrm{TM}}$ (FD Neuro Technologies Columbia, MD, USA) was used according to the manufacturer's instructions [29]. After collecting VN tissues, samples were immediately immersed in the Solution A/B for 14 $\mathrm{d}$ and then transferred to the Solution $\mathrm{C}$ for $3 \mathrm{~d}$ at room temperature protected from light. Samples were cut as a series of $150 \mu \mathrm{m}$ coronal sections via a vibratome (Leica VT 1200 S, Japan). The staining process was performed according to the manufacturer's instructions. In brief, sections were stained with the mixed working solution (Solution D: Solution E: double-distilled water $=1: 1: 2$ ) for $10 \mathrm{~min}$. After rinsing in double-distilled water for 2 times, sections were then dehydrated in a graded series of ethanol (50\%, 75\%, 95\% and $100 \%, 4$ min each concentration) and xylene ( 3 times, 4 min each time) before a coverslip with Permount ${ }^{\mathrm{TM}}$ Mounting Medium (Fisher Scientific Co., MA USA) was placed.

Glutamatergic and GABAergic neurons are two primary neural components of $\mathrm{VN}$, and glutamatergic neurons were recently considered to act as the fundamental role in controlling posturo-locomotor behaviors [30]. Thus, the excitatory pyramidal neurons in the $\mathrm{VN}$ region obtained under a microscope (Axio Imager A2, Zeiss, German) were selected for dendritic spines analysis according to previous protocol [31]. The blinded investigator analyzed images using Image-pro Plus 6.2 software 
(Bethesda, MD, USA) ( $n=3$ rats/group, 5 images for each rat).

\section{Statistical analysis}

All the data were presented as the mean \pm SD. SPSS 20.0 software (SPSS Inc., IBM, USA) and GraphPad Prism version 8.0 (GraphPad Software Inc, CA, USA) was used for statistical analysis and graph generation. Two-tailed Student's $t$-test was performed for statistical comparisons between two groups. One-way ANOVA followed by post hoc analysis with the Dunnett's test was used for statistical comparisons among groups. Two-way ANOVA with the Bonferroni post hoc test was used for behavioral data analysis. Mann-Whitney $U$ test was performed for the nonparametric analysis. The value of $p<0.05$ was considered statistically significant.

\section{Results}

\section{Repeated NTG administration induced mechanical} allodynia, thermal hyperalgesia and vestibular dysfunction We used intermittent NTG administration for 9 days as a model of CM. The model produces mechanical allodynia and thermal hyperalgesia that lasts for 7 days after final treatment [32]. The thresholds for mechanical stimulation, as well as the latencies to noxious heat were significantly reduced in a time dependent manner on day 5,7 and 9 in NTG-treated animals when compared with saline -treated animals $(p<0.05$; Fig. $1 \mathrm{~B}$ and D F). Acute hypersensitivity was evident by sustained reduced thresholds for withdrawal from mechanical $(p<0.05$; Fig. $1 \mathrm{C}$ and $1 \mathrm{E})$ and thermal stimulation (Fig. 1G). Consistent with our previous study, chronic injection of NTG produced marked vestibular dysfunction [8]. In line with the development of tactile and thermal hypersensitivity, NTG administration significantly extended the time that rats spent traversing the balance beam $(p<0.05$; Fig. $1 \mathrm{H})$ and that turn to $180^{\circ}$ upward in negative geotaxis test $(p<0.05$; Fig. 1I) on day 5, 7 and 9 when compared with the saline group and before injection data. Furthermore, the elevated expression of CGRP in the superficial layers of TNC has been considered as a key index to the development of central sensitization after CM [10,33]. Immunofluorescence staining showed that the density of CGRP-immunoreactive fibers in TNC was significantly increased in CM group compared to saline group ( $p<0.05$; Fig. $2 \mathrm{~A})$. The above data all indicated that we established a reliable rat model of $\mathrm{CM}$ that had vestibular dysfunction, which could be used in the following experiments.

\section{Endogenous CGRP, CLR and RAMP1 were upregulated in VN after CM}

First, we examined whether endogenous CGRP expression in VN would change in response to repeated NTG administration. We evaluated expression of CGRP by western blot analysis in CM and saline group. The protein level of endogenous CGRP was significantly increased after CM compared to saline group $(p<0.05$; Fig. $2 \mathrm{~B})$. Given that CGRP1 receptor complex has two functional components, we then evaluated expression levels of CLR and RAMP1 by immunofluorescence staining and qPCR in $\mathrm{CM}$ rats. Compared to the saline group, the number of CLR $+(p<0.05$; Fig. 2D upper panel and 2E left $)$ and RAMP1 $+(p<0.05$; Fig. 2D lower panel and 2E right $)$ cells increased significantly in CM rats. Similar changes have been observed in the mRNA levels of CLR and RAMP1 (Fig. 2 F), indicating that these two receptor components were activated in VN after CM. Therefore, CGRP1 receptor specific antagonist, BIBN4096BS, was selected as the main treatment after CM.

Furthermore, double immunofluorescence staining showed that CLR (Fig. 2G lower panel) and RAMP1 (Fig. $2 \mathrm{H}$ lower panel) were abundantly expressed in neurons located $\mathrm{VN}$ areas (Fig. $2 \mathrm{C}$ ) of $\mathrm{CM}$ rats. By contrast, CLR and RAMP1 seemed to have minor expression level in astrocytes (Fig. 2G H upper panel) and microglia (Fig. 2G H middle panel) in VN of CM rats (GFAP for astrocytes, Iba1 for microglia, and NeuN for neurons).

\section{Blockage of CGRP1 receptor with BIBN4096BS attenuated NTG-induced mechanical allodynia, thermal hyperalgesia and vestibular dysfunction}

To further investigate the role of CGRP1 receptor in the development of mechanical allodynia, thermal hyperalgesia and vestibular dysfunction after $C M$, we administered CGRP1 receptor antagonist BIBN4096BS (0.1 $\mu \mathrm{g} /$ day), a noncompetitive antagonist, to the lateral ventricle prior to each NTG injection. Single treatment with BIBN4096BS markedly attenuated acute mechanical allodynia and thermal hyperalgesia compared to $\mathrm{CM}+$ vehicle group $(p<0.05$; Fig. $3 \mathrm{C}$, E, and G). Multiple treatments with BIBN4096BS significantly increased basal mechanical and thermal pain thresholds when compared with $\mathrm{CM}+$ vehicle group ( $p<0.05$; Fig. 3B, D, and F). BIBN4096BS treatment significantly reversed NTG-induced vestibular dysfunction, as evidenced by less time in traversing the balance beam and negative geotaxis test compared to $\mathrm{CM}+$ vehicle group $(p<0.05$; Fig. $3 \mathrm{H}$ and $\mathrm{I})$.

\section{Inhibiting CGRP1 receptor with BIBN4096BS ameliorated overexpression of synaptic associated proteins}

In order to explore the involvement of enhanced synaptic transmission in $\mathrm{VN}$ after $\mathrm{CM}$, and the regulating role of BIBN4096BS on synaptic transmission efficiency in CM, we first analyzed several key synaptic associated proteins (post-synaptic density protein 95: PSD95; synaptophysin: Syp; synaptotagmin-1: Syt-1) in VN by western blot 


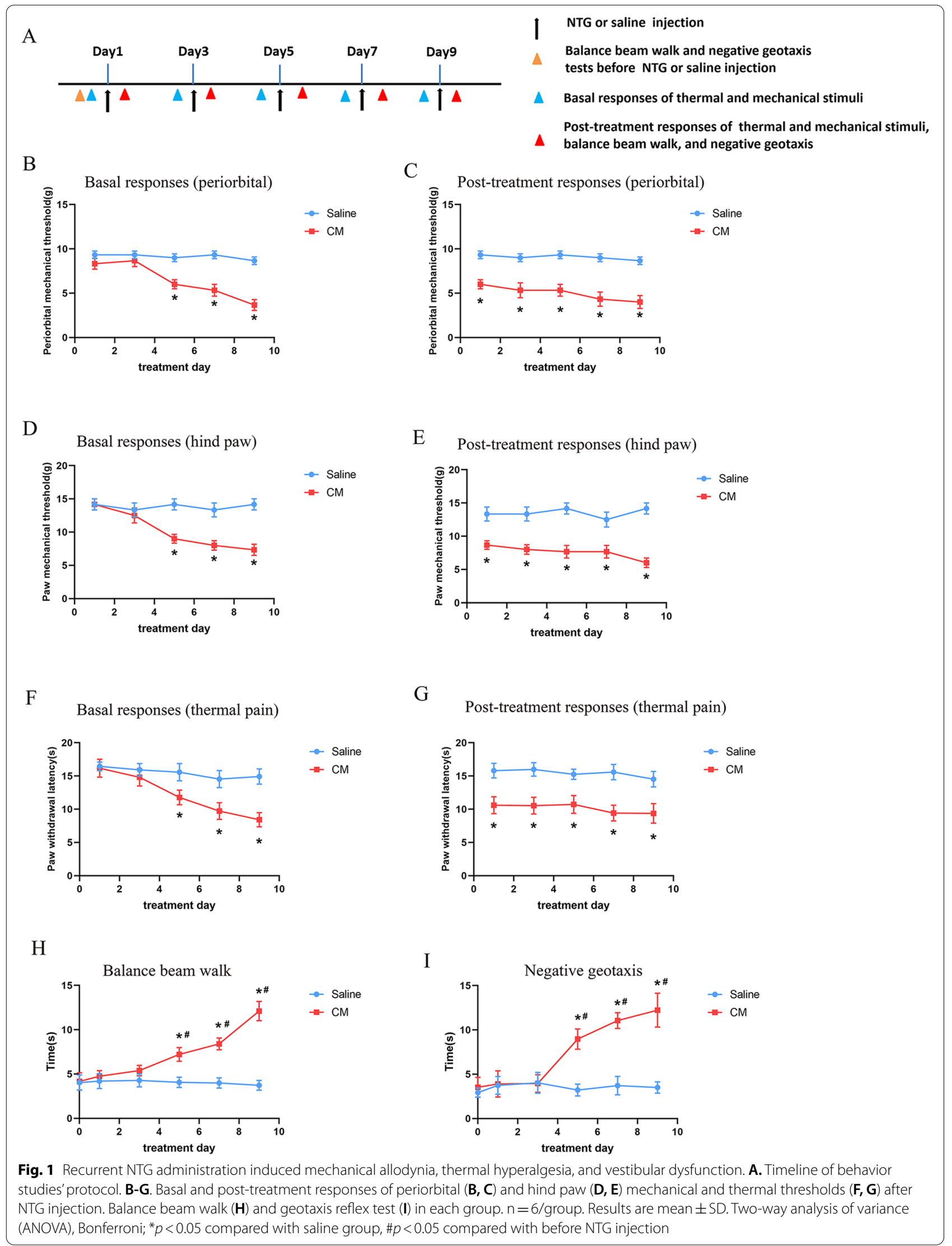




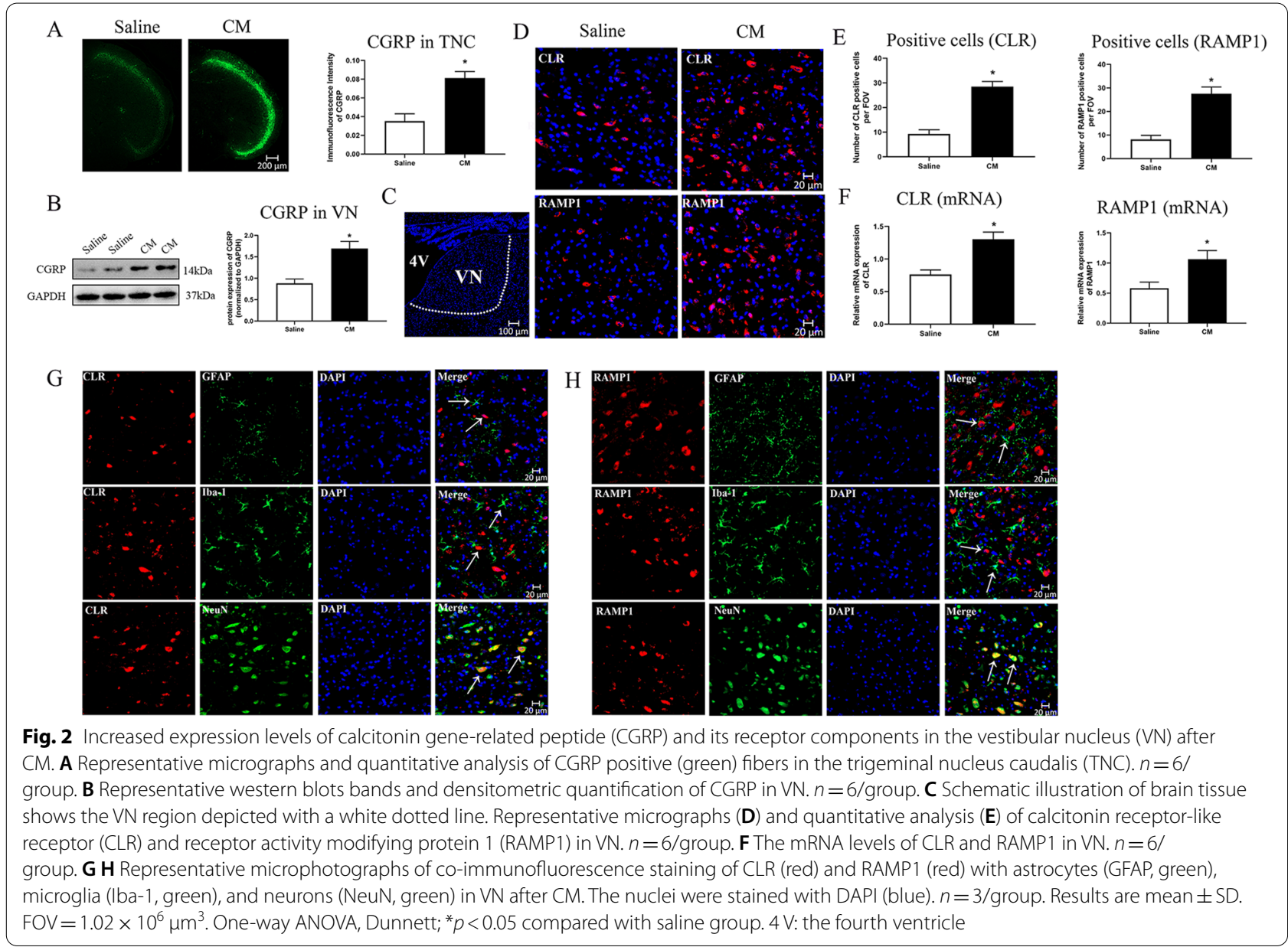

analysis. PSD95 and Syp are representative makers of postsynaptic and presynaptic respectively. And Syt-1 is an important $\mathrm{Ca}^{2+}$-sensor that strengthens synaptic connections [31]. While the expressions of PSD-95, Syp and Syt-1 were significantly increased in the CM compared to saline group $(p<0.05$; Fig. 4 A-C). Inhibiting CGRP1 receptor with BIBN4096BS markedly decreased the expression levels of PSD-95, Syp and Syt- 1 compared to the $\mathrm{CM}+$ vehicle group $(p<0.05$; Fig. 4 A-C).

\section{Inhibiting CGRP1 receptor with BIBN4096BS restored the aberrant synaptic ultrastructure and the density of neuronal dendritic spines}

The structural alterations of synapses are critical to synaptic transmission and synaptic plasticity [34]. Thus, the thickness of PSD, length of synaptic activity zone, and curvature of synaptic interface were selected as morphological indicators of synapses in this study [21]. When compared with saline group, CM significantly increased the PSD thickness $(p<0.05$; Fig. $4 \mathrm{D}$ and E), length of synaptic activity zone $(p<0.05$; Fig. $4 \mathrm{D}$ and $\mathrm{F})$, and synaptic interface curvature $(p<0.05$; Fig. $4 \mathrm{D}$ and G). After multiple BIBN4096BS treatments, these morphological alternations were significantly alleviated, and the morphological characteristics of synapses were comparable as those in saline group (Fig. 4D-G).

Furthermore, as described in previous study [31], we detected the alternation of dendritic spines in $\mathrm{VN}$, which is considered as the major postsynaptic sites for excitatory input [35]. Golgi-cox staining showed more abundant dendritic spines in $\mathrm{CM}$ and $\mathrm{CM}+$ vehicle group

(See figure on next page.)

Fig. 3 BIBN4096BS and chelerythrine chloride (CHE) treatment ameliorated mechanical allodynia, thermal hyperalgesia and vestibular dysfunction after CM. A Timeline for the treatment study. BIBN4096BS and CHE (PKC inhibitor) treatment ameliorated the decreased mechanical (B-E) and thermal (F-G) thresholds after CM. BIBN4096BS and CHE treatment alleviated vestibular dysfunction (H-I) after CM. $n=6 /$ group. Results are mean \pm SD. Two-way ANOVA, Bonferroni; ${ }^{*} p<0.05$ compared with $C M+$ vehicle group, $\# p<0.05$ compared with before NTG injection 


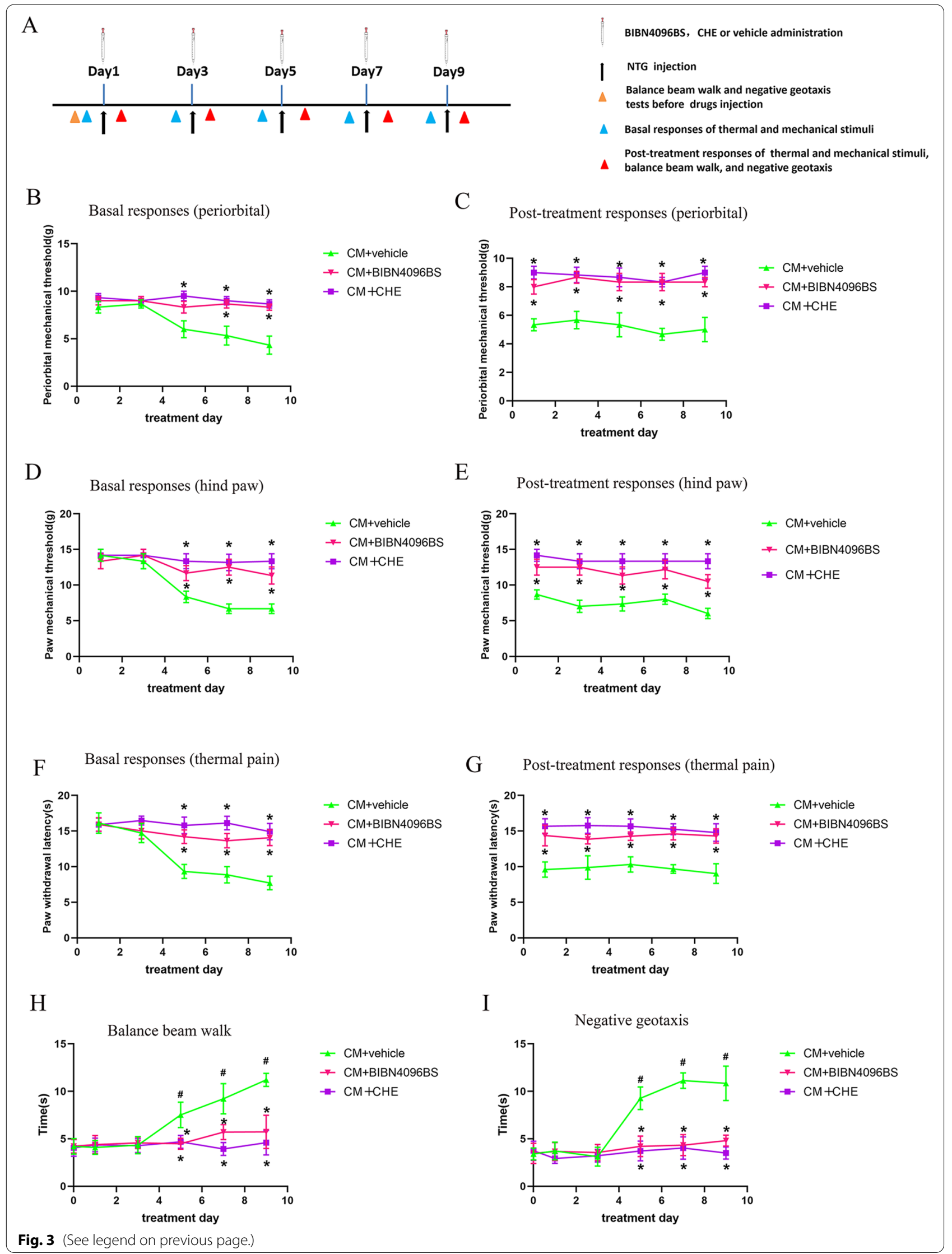


compared to saline group ( $p<0.05$; Fig. $4 \mathrm{H}$ and I). Multiple BIBN4096BS treatments significantly decreased the density of dendritic spines $(p<0.05$; Fig. $4 \mathrm{H}$ and I). These results suggested an enhanced synaptic transmission in VN after CM, while BIBN4096BS treatment could significantly abolish this enhancement.

\section{Inhibiting CGRP1 receptor with BIBN4096BS regulated the PKC/ERK/CREB signal and attenuated neuronal activation in VN after CM}

In vivo and in vitro studies show that the PKC/ERK/ CREB pathway plays an important role in CGRP related pain transmission and central sensitization $[19,20]$. However, whether the PKC/ERK/CREB signaling was a potential pathway involved in CGRP1 receptor-mediated regulation of synaptic transmission in $\mathrm{VN}$ after $\mathrm{CM}$ remains unclear. The endogenous expressions of PKC, phosphorylated ERK and CREB-S133 were significantly elevated in CM group compared to saline group $(p<0.05$; Fig. 5E-G). The addition of BIBN4096BS did not alter CGRP, CLR and RAMP1 expressions in VN after CM (Fig. 5 A-D). The expression of PKC, phosphorylation of ERK and CREB-S133 were significantly reduced in BIBN4096BS treated CM rats compared to vehicle treated CM rats $(p<0.05$; Fig. $5 \mathrm{E}-\mathrm{G})$.

Administration of CHE, the specific PKC inhibitor, to $\mathrm{CM}$ rats showed similar behavior improvements as CM+BIBN4096BS group when compared with $\mathrm{CM}+$ vehicle group (Fig. 3B-I). Treatment with CHE markedly decreased the expression levels of PSD95, Syt-1 and Syp compared to the CM+vehicle group ( $<<0.05$; Fig. 6 A-C). The expression of PKC, phosphorylation of ERK and CREB-S133 were significantly reduced in $\mathrm{CHE}$ treated $\mathrm{CM}$ rats compared to $\mathrm{CM}$ and $\mathrm{CM}+$ vehicle $(p<0.05$; Fig. $6 \mathrm{D}-\mathrm{F})$. These results support the notion that CGRP may regulate synaptic transmission through CGRP1 receptor/PKC/ERK/CREB signal.

C-Fos has been demonstrated as a classic marker for detecting neuronal activation after noxious stimulation [36]. Quantitative analysis of immunofluorescence staining found a greater density of c-Fos positive staining neurons in $\mathrm{VN}$ after $\mathrm{CM}$, and this effect was abolished by BIBN4096BS ( $p<0.05$; Fig. 5I-J). PKC inhibition showed similar tendency as BIBN4096BS administration (Fig. 6G).

\section{Discussion}

Multiple lines of evidence show that migraine and vestibular symptoms are frequently encountered clinically $[2,4,37]$. The rate of disability is high, and there is currently a lack of effective prophylactic treatment [38]. The exact pathophysiology of vestibular symptoms in migraine is incompletely understood but could include anatomical pathways and neurochemical modulation between TNC and VN $[8,39]$. CGRP or CGRP1 receptor antibodies and antagonists have proved to appreciably alleviate headache severity and frequency in both of episodic and chronic migraine patients [9]. Our previous study found that increased expression of CGRP was positively associated with neuronal activation in $\mathrm{VN}$, as well as vestibular dysfunction after CM [8]. To date, whether CGRP1 receptor involves in the development of vestibular sensitization, and how CGRP regulates neuronal activation in $\mathrm{VN}$ after $\mathrm{CM}$ remains largely unknown. Thus, the present study was to investigate whether CGRP1 receptor contributes to vestibular dysfunction after $\mathrm{CM}$ by regulating synaptic transmission in $\mathrm{VN}$.

Our results showed that CGRP and two major components of its receptor, CLR and RAMP1, were significantly elevated in VN after CM. CLR and RAMP1 were primarily co-expressed with neurons in VN. Multiple treatments with CGRP1 receptor selective antagonist, BIBN4096BS, alleviated mechanical allodynia, thermal hyperalgesia and vestibular dysfunction induced by intermittent administration of NTG, and this process was associated with the downregulation of synaptic associated proteins, restoration of aberrant morphological characteristics of synapses, and suppression of neuronal activation in VN after CM. Inhibiting CGRP1 receptor with BIBN4096BS decreased the overexpression of PKC, and phosphorylation of ERK and CREBS133 in VN after CM. Taken together, our data suggests the therapeutic potentials of BIBN4096BS to restore vestibular dysfunction in patients with CM.

Consistent with our previous study, we established a reliable $\mathrm{CM}$ model that had vestibular dysfunction via systematic NTG administration [8]. Each NTG administration caused several behavior signs of allodynia and hyperalgesia, mimicking the acute attack of migraine. While repeated NTG administration induced

\footnotetext{
(See figure on next page.)

Fig. 4 BIBN4096BS treatment reduced overexpression of synaptic associated proteins and restored the aberrant synaptic ultrastructure in VN after CM. Representative western blot bands and densitometric quantification of PSD-95 (A), Syp (B) and Syt-1 (C) after CM. $n=6 / \mathrm{group}$. D Representative images of the synaptic ultrastructure in VN after CM. Quantitative analysis of PSD thickness (E), length of synaptic activity zone $(\mathbf{F})$, and curvature of synaptic interface $(\mathbf{G})$ after $C M . n=3$ /group. Representative images $(\mathbf{H})$ and quantitative analysis $(\mathbf{I})$ of the density of dendritic spines in VN after CM. $n=3 /$ group. Results are mean \pm SD. One-way ANOVA, Dunnett; ${ }^{*} p<0.05$ compared with saline group, \#p<0.05 compared with $\mathrm{CM}+$ vehicle group
} 

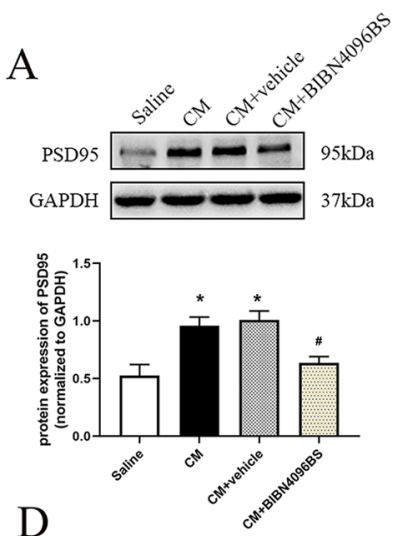

Saline

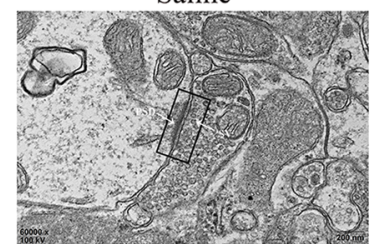

$\mathrm{CM}+$ vehicle

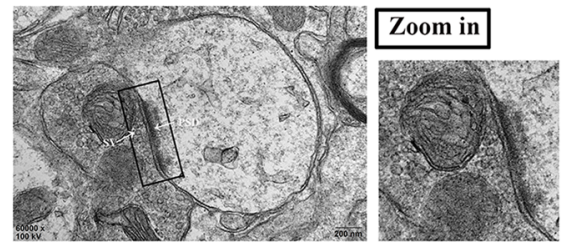

E

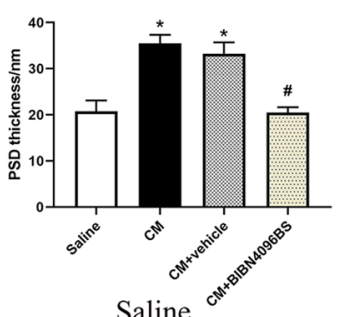

$\mathrm{H}$

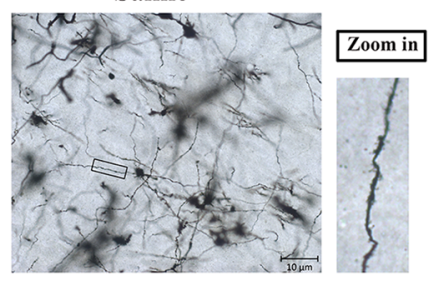

$\mathrm{CM}+$ vehicle

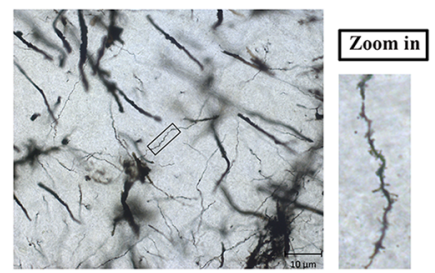

$\mathrm{F}$
B
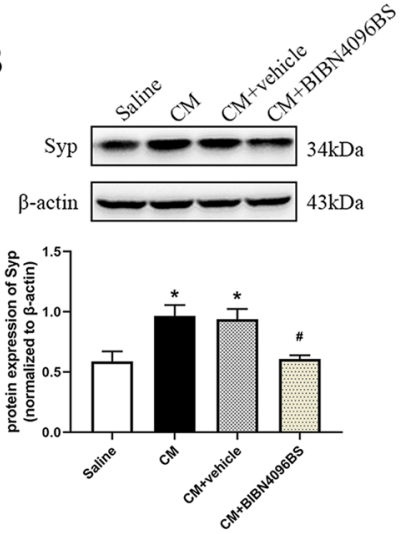

C
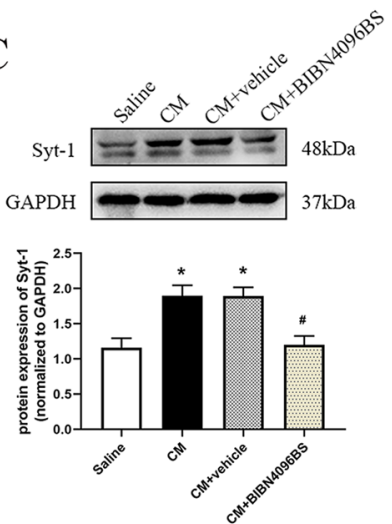
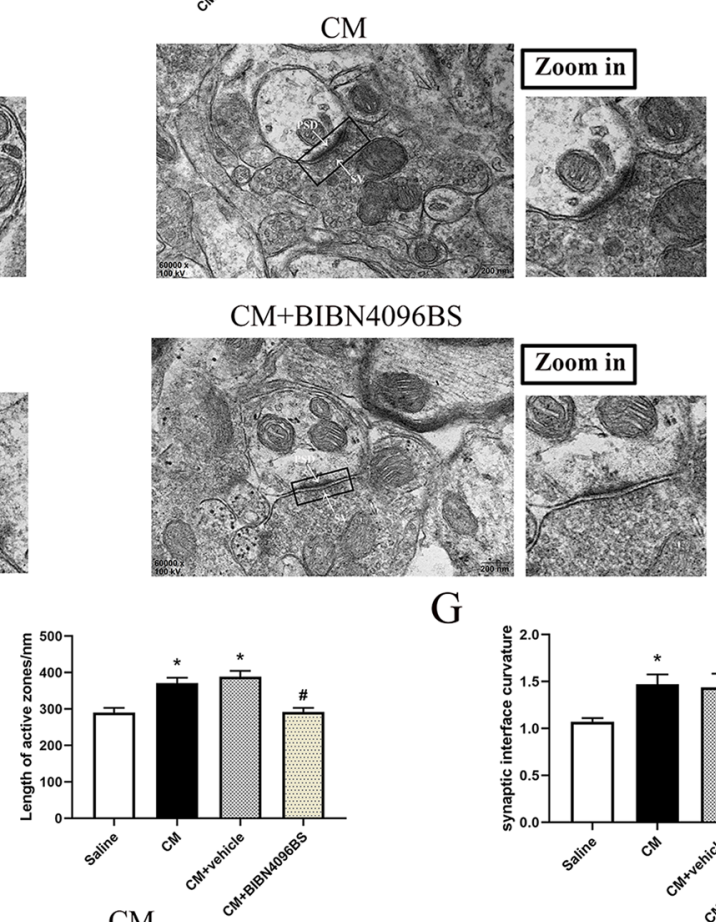

G
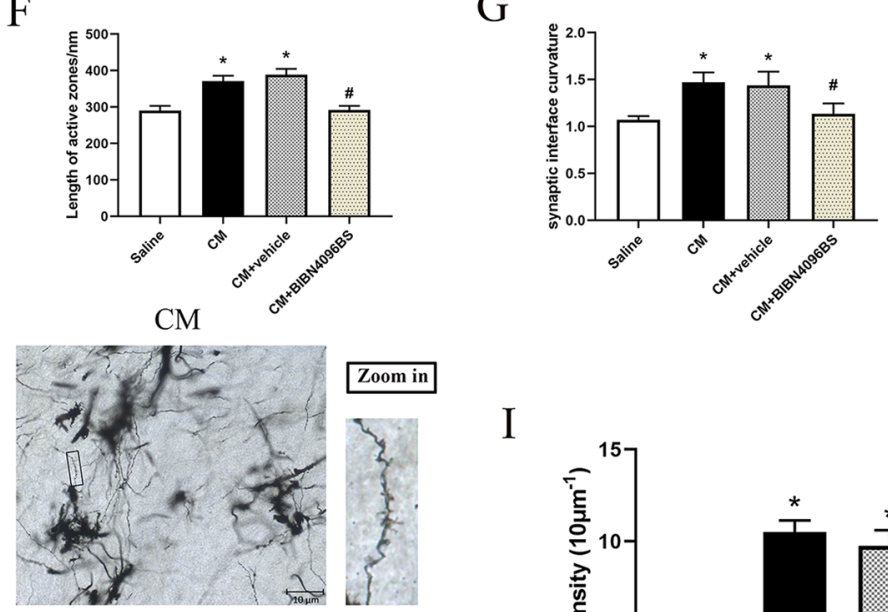

CM+BIBN4096BS

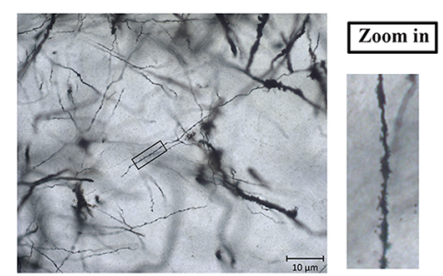

I

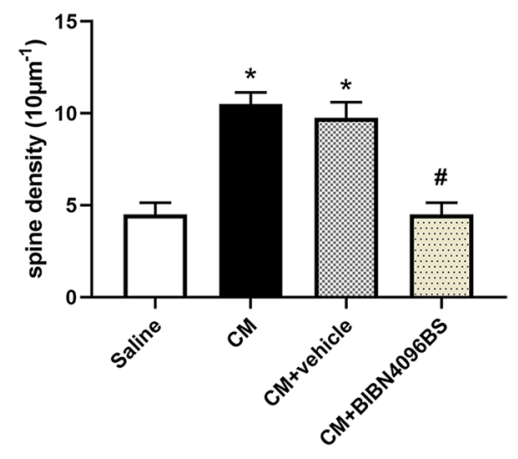




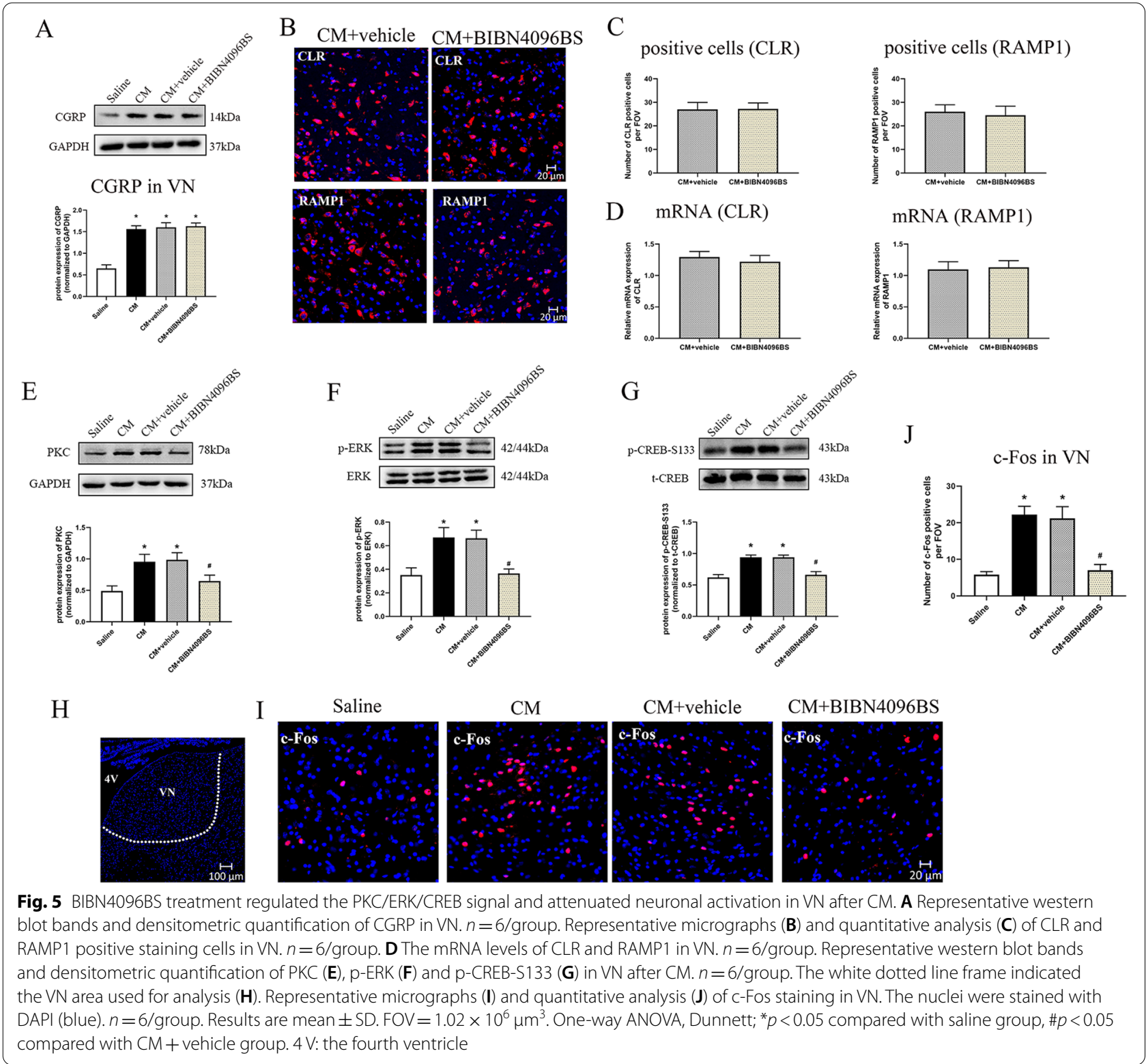

the pronounced and sustained allodynia and hyperalgesia as found in most of CM patients [10, 32]. Unlike the lowered pain thresholds, significant vestibular dysfunction was not observed after the initial NTG injection, and vestibular function was exaggerated after the third NTG injection. These phenomena were consistent with those reported in clinical-based studies: the development of vestibular symptoms, including motion intolerance, often lagged several years behind headache in migraine patients; patients with chronic migraine experienced more frequent vestibular symptoms than patients with episodic migraine [2, 40, 41]. Additionally, migraine is more prevalent in women than in men, which are mainly due to the role of estrogen $[42,43]$. In fact, the regulation of pain by estrogen is very complicated. The level and stability of estrogen can affect its modulation of pain, including aggravating or relieving [44]. The main trigger factors of menstrual-related migraine seem to be the withdrawal of estrogen, and reducing the magnitude of decline in estrogen concentration can prevent menstrual-related migraine [43, 45]. Studies have found that endogenous changes in the concentration of estrogen and progesterone in premenstrual syndrome or the use of exogenous hormones (such as oral contraceptives) may cause vertigo [46]. In order to avoid the influence of estrogen, we chose male mice to establish the model. 

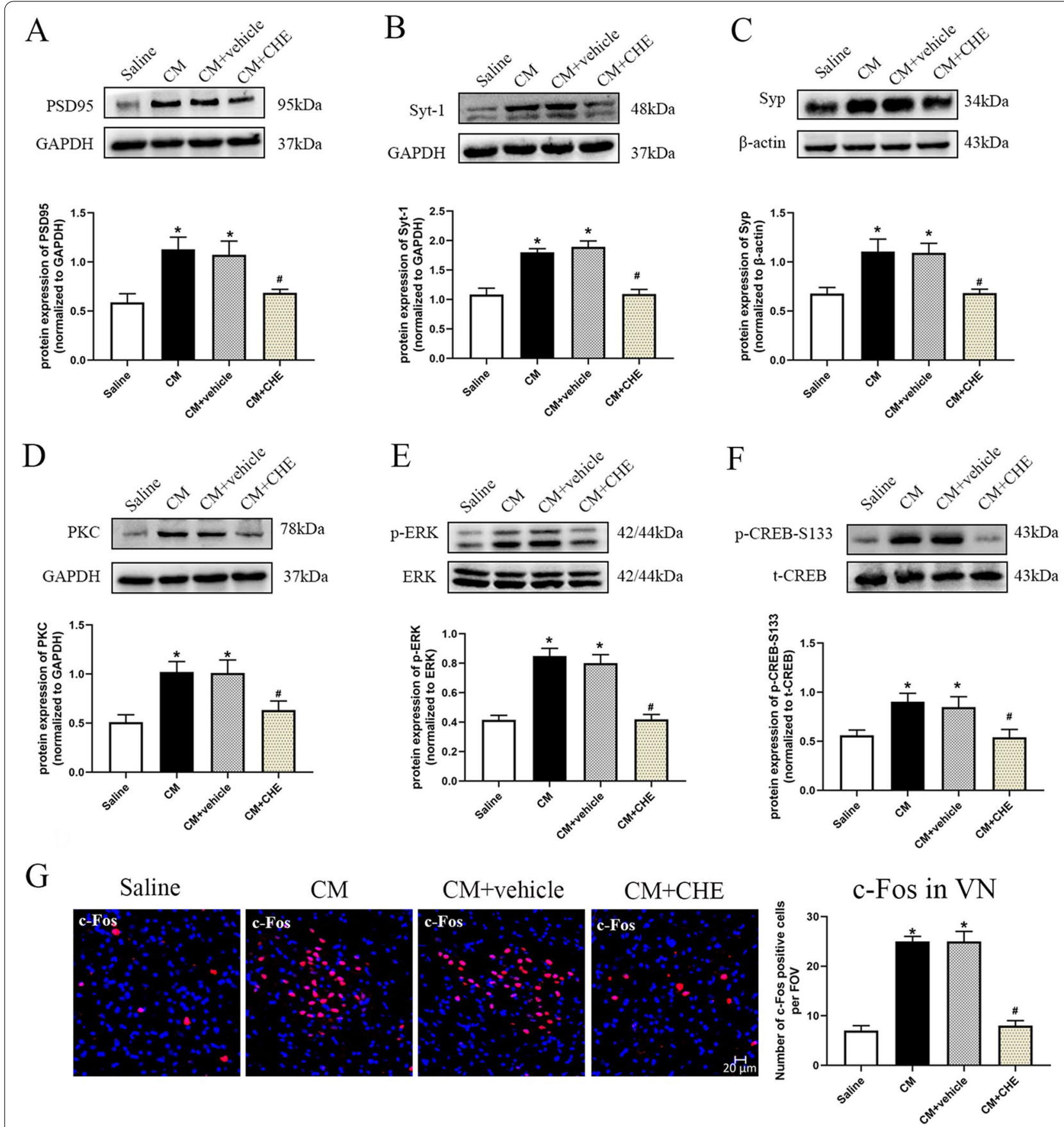

Fig. 6 Inhibition of PKC activity suppressed NTG-induced overexpression of synaptic associated proteins, reduced downstream phosphorylated ERK and CREB-S133 levels and inhibited neuronal activation after CM. Representative western blot bands and densitometric quantification of PSD-95 (A), Syt-1 (B), Syp (C), PKC (D), p-ERK (E) and p-CREB-133 (F) after CM. $n=6 /$ group. G. Representative micrographs and quantitative analysis of c-Fos staining in VN. The nuclei were stained with DAPI (blue). $n=6 /$ group. Results are mean \pm SD. FOV $=1.02 \times 10^{6} \mu m^{3}$. One-way ANOVA, Dunnett; ${ }^{*} p<0.05$ compared with saline group, $\# p<0.05$ compared with $C M+$ vehicle group

Given the significant sex differences in the prevalence of migraine, the sex dimorphism of CGRP1 receptor involvement in pathogenesis of migraine-related dizziness requires further exploration.
A plethora of studies have demonstrated the critical role of CGRP in the trigeminovascular system that are involved in migraine pathology [47]. Abundant expression of CGRP in laminae I and II of the TNC has been 
reported as an important indicator for central sensitization of CM, which is responsible for the development of spontaneous cephalic cutaneous and extracephalic allodynia [5, 21]. Consistently, this study also observed the elevated expression of CGRP in TNC and VN after CM. In addition, reducing CGRP expression in $\mathrm{VN}$ attenuated excitability of neurons and reversed the vestibular dysfunction induced by NTG administration [8]. These data indicated that elevated CGRP level in VN might be an index to vestibular sensitization in CM rats. The various effects of CGRP act primarily through CGRP1 receptor, which comprising CLR and RAMP1 $[47,48]$. In preclinical animal models and clinical trials, "gepants" are proved to have high affinity for CGRP1 receptor [48]. To make it easier to translate our findings for clinical application, the present study applied BIBN4096BS (namely: olcegepant) as the treatment regimen [48, 49]. However, another GPCR, the calcitonin receptor (CTR), which is closely-related to CLR, together with RAMP1 (namely: $\mathrm{AMY}_{1}$ receptor) recently has been reported to have high affinity for both of CGRP and amylin [48]. Additionally, adrenomedullin, an amylin analogue, is demonstrated to effectively provoke migraine-like headache in migraine patients [50]. Less is known about the nociceptive role of $\mathrm{AMY}_{1}$ receptor in migraine pathology and the clinical significance of the interaction between CGRP and AMY receptor. Thus, we selected CGRP1 receptor as the primary receptor for the following studies.

CGRP1 receptor are broadly expressed in the mammalian CNS [5, 51]. Considering the limited passage of the gepants (2\%) across the blood-brain barrier, BIBN4096BS was administrated via intracerebroventricular route to explore the central action of CGRP1 receptor [47]. We found that antagonizing CGRP1 receptor significantly abolished behavioral signs of mechanical allodynia, thermal hyperalgesia and vestibular dysfunction in CM rats, and these were closely related with the neuronal activation in VN. But how CGRP modulates the neuronal activation in $\mathrm{VN}$ after $\mathrm{CM}$ is still unclear. According to the immunofluorescence staining, CLR and RAMP1 were both primarily co-expressed with neurons, implying that CGRP might exert its effect mainly through the neuronal CGRP1 receptor. In vivo and in vitro studies demonstrated that exogenous CGRP can enhance the neural synaptic transmission in spinal cord, anterior cingulate cortex and insular cortex, and that could be notably blocked by BIBN4096BS $[15,16,52,53]$. Therefore, we proposed that CGRP could modulate the synaptic transmission efficiency via CGRP1 receptor in VN after CM. We first examined the expression of several synaptic associated proteins, including PSD95, Syp and Syt-1, in VN after CM. PSD95 is a structural protein located on the postsynaptic membrane, implicated in synaptic maturation, strengthening, and plasticity [54]. Syp, a major integral membrane protein of synaptic vesicles, is necessary for vesicle formation and exocytosis, which can regulate the efficiency of synaptic transmission by influencing the synaptic structure and the release of neurotransmitters [55, 56]. Syt- 1 , acting as a $\mathrm{Ca}^{2+}$ sensor at neuronal synapses, is a marker of synaptic transmission, which synchronizes neurotransmitter release with $\mathrm{Ca}^{2+}$ influx during action potential firing and mediates ultrafast exocytosis of synaptic vesicles $[57,58]$. Our study found that the expression of synaptic associated proteins was significantly increased after CM, while multiple treatments of BIBN4096BS markedly downregulated their expression. Moreover, we detected the synaptic ultrastructure and the density of neuronal dendritic spines of $\mathrm{VN}$ neurons in $\mathrm{CM}$ rats, which are closely related to synaptic transmission efficiency, to further confirm that CGRP/CGRP1 receptor involves in the regulation of synaptic transmission in $\mathrm{VN}$ after $\mathrm{CM}$.

We further explored downstream orchestrators of CGRP1 receptor inhibition to determine potential mechanisms for neuronal activation. Activation of the glutamate $\mathrm{N}$-methyl-D-aspartate (NMDA) receptors is a hallmark to the development and maintenance of central sensitization in chronic pain, including migraine [10]. Incubation with CGRP or activation of CGRP1 receptor significantly promotes NMDA receptors-mediated excitatory potentials in central amygdala $[10,59]$. The downstream activation of PKC induces phosphorylation of NMDA receptors and increases calcium inputs which further enhances the excitability of dorsal horn neuron, thus maintaining a state of central sensitization $[10,19]$. Similar to the previous results, we found that the expression levels of PKC, phosphorylated ERK and CREB-S133 were significantly increased in CM rats. The effects of NTG administration on PKC expression and phosphorylation of ERK and CREB-S133 were reversed by BIBN4096BS. Additionally, inhibition of PKC with $\mathrm{CHE}$ reduced the overexpression of synaptic associated proteins, p-ERK and p-CREB-S133 caused by CM, and inhibited the activation of neurons in the $\mathrm{VN}$ of $\mathrm{CM}$ rats. These results suggested that CGRP may improve synaptic transmission and neuron activation of $\mathrm{VN}$ through CGRP1 receptor/PKC/ERK/CREB in CM rats.

In neuronal circuits, the vast majority of excitatory synaptic transmission occurs at the postsynaptic dendritic spines [60]. In our studies, we found more abundant dendritic spines in CM group compared to saline group. And multiple BIBN4096BS treatments significantly decreased the density of dendritic spines in VN. Protein kinase C (PKC)-dependent mechanisms can promote synaptic function in the mature brain. Studies found that the activation of protein kinase $\mathrm{C}$ (PKC) $\varepsilon$ and $\alpha$ has been linked 
to synaptogenesis. And $\mathrm{PKC} \varepsilon / \alpha$ activator bryostatin can increase dendritic spine density of pyramidal neurons in hippocampal CA1 region of aged rats [61]. In mature mouse hippocampal neuron cultures, PKC activator bryostatin increased the proportion of mushroom dendritic spines in total spine density, enhanced synaptic transmission [62]. As well, atypical protein kinase C (aPKC), a subgroup of $\mathrm{PKC}$, can promote increased dendritic spine density in hippocampal neurons through p190 RhoGAP and RhoA pathway $[63,64]$. In our research, we found that inhibiting $\mathrm{PKC}$ with chelerythrine chloride $(\mathrm{CHE})$ ameliorated the overexpression of synapse-associated proteins. Therefore, we speculate that the activation of PKC pathway can also cause an increase in the density of neuronal dendritic spines at the VN site. More direct evidence is needed in our future studies.

According to previous research, under physiological conditions, the GABAergic neurons that project to the vestibular nucleus are mainly located in spinal trigeminal nucleus orails (Sp5O) and a small amount of spinal trigeminal nucleus interpolaris (Sp5I), and the glutamatergic neurons that project to $\mathrm{VN}$ are mainly located at $\mathrm{Sp} 5 \mathrm{O}, \mathrm{Sp} 5 \mathrm{I}$ and spinal trigeminal nucleus candalis (Sp5C or TNC). GABA immunoreactive neurons account for about $15 \%$ of trigeminal vestibular neurons, and glutamatergic neurons account for about $37 \%$ of the trigeminal vestibular neurons [39]. Our previous study found that $\mathrm{CM}$ induced neuronal activation in the TNC and $\mathrm{VN}$, and TNC-projecting $\mathrm{VN}$ neurons were activated after CM [8]. As well, glutamatergic neurons of $\mathrm{VN}$, the excitatory neurons, were recently considered to act as the fundamental role in controlling posturo-locomotor behaviors [30]. However, we currently have no direct evidence for the type of neurons activated at the $\mathrm{VN}$ site in CM rats. We will explore it more deeply in future research.

There are several limitations in this study. Although CGRP1 receptor is mainly expressed in neurons of VN areas, we cannot completely exclude that the neuroprotective effects exerted by CGRP1 receptor inhibition may involve glia in $\mathrm{CM}$ rats. Also, we are focusing on $\mathrm{PKC} /$ ERK/CREB signaling, but other mechanisms require further investigation. Then, we didn't perform the basal vestibular function test before each NTG administration. Although we speculate that vestibular dysfunction in migraine patients may be a progressive rather than acute process, the data of the vestibular function test before each NTG administration may help us understand the development of the disease comprehensively. Our research ignored the study of three synaptic associated proteins in the synaptic subcellular fractions, and we will pay attention to this part in the future research. In our study, BIBN4096BS and protein kinase C (PKC) inhibitor chelerythrine chloride (CHE) were administered intracerebroventricularly, rather than local microinjection into the VN. Therefore, we cannot completely rule out effects of these drugs on other brain regions. Last, we only explored the effects of morphological alternations on synaptic transmission. Future studies on electrophysiology are needed to examine the effects on synaptic function.

\section{Conclusions}

In conclusion, our results demonstrated that CGRP/ CGRP1 receptor involved in the morphological alternation of synapses in $\mathrm{VN}$ and influenced vestibular function in an experimental CM rat model, at least partly, through PKC/ERK/CREB pathway. Most importantly, we also demonstrated that BIBN4096BS, a specific CGRP1 receptor antagonist, restored the synaptic ultrastructure, attenuated enhancement of neuronal activity and alleviated mechanical allodynia, thermal hyperalgesia and vestibular dysfunction in CM rats. Our findings may provide new strategies for the treatment of migraine patients with vestibular symptoms.

\section{Abbreviations \\ ANOVA: Analysis of variance; ARRIVE: Animal Research Reporting of In Vivo Experiments; CGRP: Calcitonin Gene-related Peptide; CLR: Calcitonin Receptor- like Receptor; CREB: cAMP Response Element-binding Protein; CM: Chronic Migraine; CHE: Chelerythrine Chloride; ERK: Extracellular Signal Regulated Kinase; FOV: Field of View; GPCR: G-protein-coupled Receptor; NMDA: Gluta- mate N-methyl-D-aspartate; NTG: Nitroglycerin; PKC: Protein Kinase C; PSD95: Post-synaptic Density Protein 95; PSD: Postsynaptic Density; qPCR: Quantita- tive Real-time Polymerase Chain Reaction; RAMP1: Receptor Activity Modifying Protein 1; Syp: Synaptophysin; Syt-1: Synaptotagmin-1; Sp50: trigeminal nucleus orails; Sp5l: trigeminal nucleus interpolaris; Sp5C: trigeminal nucleus candalis; TNC: Trigeminal Nucleus Caudalis; VN: Vestibular Nucleus.}

\section{Acknowledgements}

Not applicable.

\section{Authors' contributions}

This study was designed by RT, YZ, YXZ, and JZ. The experiments were completed by RT, YZ, QP, YW, QW, XF, GQ, and DZ. RT and $Y Z$ performed statistical analysis. RT, YXZ and JZ finished writing the manuscript. YXZ, LC and JZ provided supervision and final check. All the authors read the final version of this paper and approved it.

\section{Funding}

This study was supported by the National Natural Science Foundation of China (No: 82101296 to Yixin Zhang) and the Cultivation Fund of The First Affiliated Hospital of Chongqing Medical University (NO: ZYRC2020-01 to Yixin Zhang).

\section{Availability of data and materials}

The data used and analyzed in this article are available from on reasonable request.

\section{Declarations}

Ethics approval

All animal experiments performed in this study were approved by the Ethics Committee for Animal Experimentation of Chongqing Medical University. 


\section{Consent for publication \\ Not applicable.}

\section{Competing interests}

The authors declare that they have no competing interests.

\section{Author details}

'Department of Neurology, The First Affiliated Hospital of Chongqing Medical University, 1st You Yi Road, Yu Zhong District, 400016 Chongqing, China.

${ }^{2}$ Department of Neurology, Nanchong Central Hospital, Nanchong, China. ${ }^{3}$ Laboratory Research Center, The First Affiliated Hospital of Chongqing Medical University, Chongqing, China.

\section{Received: 9 January 2022 Accepted: 15 February 2022} Published online: 08 March 2022

\section{References}

1. Pan Q, Zhang Y, Long T et al (2018) Diagnosis of Vertigo and Dizziness Syndromes in a Neurological Outpatient Clinic. Eur Neurol 79:287-294. Doi https://doi.org/10.1159/000489639

2. Carvalho GF, Vianna-Bell FH, Florencio LL et al (2019) Presence of vestibular symptoms and related disability in migraine with and without aura and chronic migraine. Cephalalgia: an international journal of headache 39:29-37. Doi https://doi.org/10.1177/0333102418769948

3. Vuralli D, Yildirim F, Akcali DT et al (2018) Visual and Postural MotionEvoked Dizziness Symptoms Are Predominant in Vestibular Migraine Patients. Pain Med 19:178-183. Doi https://doi.org/10.1093/pm/pnx182

4. Huang TC, Wang SJ, Kheradmand A (2020) Vestibular migraine: An update on current understanding and future directions. Cephalalgia: an international journal of headache 40:107-121. Doi https://doi.org/10.1177/ 0333102419869317

5. Goadsby PJ, Holland PR, Martins-Oliveira M et al (2017) Pathophysiology of Migraine: A Disorder of Sensory Processing. Physiol Rev 97:553-622. Doi https://doi.org/10.1152/physrev.00034.2015

6. Latremoliere A, Woolf CJ (2009) Central sensitization: a generator of pain hypersensitivity by central neural plasticity. J pain 10:895-926. Doi https://doi.org/10.1016/j.jpain.2009.06.012

7. Su M, Yu S (2018) Chronic migraine: A process of dysmodulation and sensitization. Mol Pain 14:2070395823. Doi https://doi.org/10.1177/17448 06918767697

8. Zhang Y, Zhang Y, Tian K et al (2020) Calcitonin gene-related peptide facilitates sensitization of the vestibular nucleus in a rat model of chronic migraine. J Headache Pain 21:72. Doi https://doi.org/10.1186/ s10194-020-01145-y

9. Ashina M, Hansen JM, Do TP et al (2019) Migraine and the trigeminovascular system-40 years and counting. Lancet Neurol 18:795-804. Doi https://doi.org/10.1016/S1474-4422(19)30185-1

10. lyengar S, Ossipov MH, Johnson KW (2017) The role of calcitonin generelated peptide in peripheral and central pain mechanisms including migraine. Pain 158:543-559. Doi https://doi.org/10.1097/j.pain.00000 00000000831

11. Russell FA, King R, Smillie SJ et al (2014) Calcitonin gene-related peptide: physiology and pathophysiology. Physiol Rev 94:1099-1142. Doi https:// doi.org/10.1152/physrev.00034.2013

12. Walker CS, Conner AC, Poyner DR et al (2010) Regulation of signal transduction by calcitonin gene-related peptide receptors. Trends Pharmacol Sci 31:476-483. Doi https://doi.org/10.1016/j.tips.2010.06.006

13. Xiaocheng W, Zhaohui S, Junhui X et al (2012) Expression of calcitonin gene-related peptide in efferent vestibular system and vestibular nucleus in rats with motion sickness. PLoS ONE 7:e47308. Doi https://doi.org/10. 1371/journal.pone.0047308

14. Charles A, Pozo-Rosich P (2019) Targeting calcitonin gene-related peptide: a new era in migraine therapy. Lancet (London England) 394:17651774. Doi https://doi.org/10.1016/S0140-6736(19)32504-8

15. Liu Y, Chen QY, Lee JH et al (2020) Cortical potentiation induced by calcitonin gene-related peptide (CGRP) in the insular cortex of adult mice. Mol Brain 13:36. Doi https://doi.org/10.1186/s13041-020-00580-x

16. Li XH, Matsuura T, Liu RH et al (2019) Calcitonin gene-related peptide potentiated the excitatory transmission and network propagation in the anterior cingulate cortex of adult mice. Mol Pain 15:2069330802. Doi https://doi.org/10.1177/1744806919832718

17. Han JS, Li W, Neugebauer V (2005) Critical role of calcitonin gene-related peptide 1 receptors in the amygdala in synaptic plasticity and pain behavior. J neuroscience: official J Soc Neurosci 25:10717-10728. Doi https://doi.org/10.1523/JNEUROSCI.4112-05.2005

18. Cottrell GS (2019) CGRP Receptor Signalling Pathways. Handb Exp Pharmacol 255:37-64 Doi 10.1007/164_2018_130

19. Yarwood RE, Imlach WL, Lieu T et al (2017) Endosomal signaling of the receptor for calcitonin gene-related peptide mediates pain transmission. P Natl Acad Sci Usa 114:12309-12314. Doi https://doi.org/10.1073/pnas. 1706656114

20. Mitsikostas DD, Knight YE, Lasalandra M et al (2011) Triptans attenuate capsaicin-induced CREB phosphorylation within the trigeminal nucleus caudalis: a mechanism to prevent central sensitization? J Headache Pain 12:411-417. Doi https://doi.org/10.1007/s10194-011-0352-2

21. Niu Y, Zeng $X$, Zhao L et al (2020) Metabotropic glutamate receptor 5 regulates synaptic plasticity in a chronic migraine rat model through the PKC/NR2B signal. J Headache Pain 21:139. Doi https://doi.org/10.1186/ s10194-020-01206-2

22. Long T, He W, Pan Q et al (2018) Microglia P $2 \times 4$ receptor contributes to central sensitization following recurrent nitroglycerin stimulation. J Neuroinflamm 15:245. Doi https://doi.org/10.1186/s12974-018-1285-3

23. Zeng X, Niu Y, Qin G et al (2020) Deficiency in the function of inhibitory interneurons contributes to glutamate-associated central sensitization through GABABR2-SynCAM1 signaling in chronic migraine rats. FASEB journal: official publication of the Federation of American Societies for Experimental Biology 34:14780-14798. Doi https://doi.org/10.1096/f.202001561R

24. Wang Z, Ma W, Chabot JG et al (2010) Calcitonin gene-related peptide as a regulator of neuronal CaMKII-CREB, microglial p38-NFKB and astroglial ERK-Stat $1 / 3$ cascades mediating the development of tolerance to morphine-induced analgesia. Pain 151:194-205. Doi https://doi.org/10. 1016/j.pain.2010.07.006

25. Zhou H, Wang X, Wang S et al (2019) Inhibition of Nerve Growth Factor Signaling Alleviates Repeated Dural Stimulation-induced Hyperalgesia in Rats. Neuroscience 398:252-262. Doi https://doi.org/10.1016/j.neuroscien ce.2018.12.006

26. Zhang J, Li B, Yu L et al (2011) A role for orexin in central vestibular motor control. Neuron 69:793-804. Doi https://doi.org/10.1016/j.neuron.2011. 01.026

27. Motz BA, Alberts JR (2005) The validity and utility of geotaxis in young rodents. Neurotoxicol Teratol 27:529-533. Doi https://doi.org/10.1016/j. ntt.2005.06.005

28. Ramirez RL, Spear LP (2010) Ontogeny of ethanol-induced motor impairment following acute ethanol: assessment via the negative geotaxis reflex in adolescent and adult rats. Pharmacol Biochem Behav 95:242-248. Doi https://doi.org/10.1016/j.pbb.2010.01.013

29. Zhou X, Liang J, Wang J et al (2020) Up-regulation of astrocyte excitatory amino acid transporter 2 alleviates central sensitization in a rat model of chronic migraine. J Neurochem 155:370-389. Doi https://doi.org/10. 1111/jnc. 14944

30. Montardy Q, Wei M, Liu X et al (2021) Selective optogenetic stimulation of glutamatergic, but not GABAergic, vestibular nuclei neurons induces immediate and reversible postural imbalance in mice. Prog Neurobiol: 102085 Doi. https://doi.org/10.1016/j.pneurobio.2021.102085

31. Wang XY, Zhou HR, Wang S et al (2018) NR2B-Tyr phosphorylation regulates synaptic plasticity in central sensitization in a chronic migraine rat model. J Headache Pain 19:102. Doi https://doi.org/10.1186/ s10194-018-0935-2

32. Pradhan AA, Smith ML, McGuire B et al (2014) Characterization of a novel model of chronic migraine. Pain 155:269-274. Doi https://doi.org/10. 1016/j.pain.2013.10.004

33. Edvinsson $L$ (2017) The Trigeminovascular Pathway: Role of CGRP and CGRP Receptors in Migraine. Headache: 47-55 Doi https://doi.org/10. 1111/head.13081

34. Rollenhagen A, Lübke JH (2006) The morphology of excitatory central synapses: from structure to function. Cell Tissue Res 326:221-237. Doi https://doi.org/10.1007/s00441-006-0288-z

35. Jaworski J, Kapitein LC, Gouveia SM et al (2009) Dynamic microtubules regulate dendritic spine morphology and synaptic plasticity. Neuron 61:85-100. Doi https://doi.org/10.1016/j.neuron.2008.11.013 
36. Harriott AM, Strother LC, Vila-Pueyo M et al (2019) Animal models of migraine and experimental techniques used to examine trigeminal sensory processing. J Headache Pain 20:91. Doi https://doi.org/10.1186/ s10194-019-1043-7

37. Iljazi A, Ashina H, Lipton RB et al (2020) Dizziness and vertigo during the prodromal phase and headache phase of migraine: A systematic review and meta-analysis. Cephalalgia: an international journal of headache 40:1095-1103. Doi https://doi.org/10.1177/0333102420921855

38. Li V, McArdle H, Trip SA (2019) Vestibular migraine. BMJ (Clinical research ed. 366:14213. https://doi.org/10.1136/bmj.14213

39. Diagne M, Valla J, Delfini C et al (2006) Trigeminovestibular and trigeminospinal pathways in rats: retrograde tracing compared with glutamic acid decarboxylase and glutamate immunohistochemistry. J Comp Neurol 496:759-772. Doi https://doi.org/10.1002/cne.20964

40. Zhang Y, Kong Q, Chen J et al (2016) International Classification of Headache Disorders 3rd edition beta-based field testing of vestibular migraine in China: Demographic, clinical characteristics, audiometric findings and diagnosis statues. Cephalalgia: an international journal of headache 36:240-248. Doi https://doi.org/10.1177/0333102415587704

41. Radtke A, Neuhauser H, von Brevern M et al (2011) Vestibular migrainevalidity of clinical diagnostic criteria. Cephalalgia: an international journal of headache 31:906-913. Doi https://doi.org/10.1177/0333102411405228

42. Brandes $J \mathrm{~L}$ (2006) The influence of estrogen on migraine: a systematic review. JAMA 295:1824-1830. Doi https://doi.org/10.1001/jama.295.15. 1824

43. Chai NC, Peterlin BL, Calhoun AH (2014) Migraine and estrogen. Curr Opin Neurol 27:315-324. Doi https://doi.org/10.1097/WCO.0000000000000091

44. Craft RM (2007) Modulation of pain by estrogens. Pain: S3-S12 Doi https://doi.org/10.1016/j.pain.2007.09.028

45. Silberstein SD (2000) Sex hormones and headache. 4S-30S, Rev Neurol-France

46. Rybak LP (1995) Metabolic disorders of the vestibular system. Otolaryngology-head and neck surgery: official journal of American Academy of Otolaryngology-Head and Neck Surgery 112: 128-132 Doi https://doi. org/10.1016/S0194-59989570312-8

47. Edvinsson L, Haanes KA, Warfvinge $K$ et al (2018) CGRP as the target of new migraine therapies - successful translation from bench to clinic. Nat reviews Neurol 14:338-350. Doi https://doi.org/10.1038/ s41582-018-0003-1

48. Garelja ML, Walker CS, Hay DL (2021) CGRP receptor antagonists for migraine. Are they also AMY receptor antagonists? Brit J Pharmacol Doi. https://doi.org/10.1111/bph.15585

49. Walker CS, Raddant AC, Woolley MJ et al (2018) CGRP receptor antagonist activity of olcegepant depends on the signalling pathway measured. Cephalalgia: an international journal of headache 38:437-451. Doi https://doi.org/10.1177/0333102417691762

50. Ghanizada H, Al-Karagholi MA, Arngrim N et al (2021) Effect of Adrenomedullin on Migraine-Like Attacks in Patients With Migraine: A Randomized Crossover Study. Neurology 96:e2488-e2499. Doi https://doi.org/ 10.1212/WNL.0000000000011930

51. Warfvinge $K$, Edvinsson L (2019) Distribution of CGRP and CGRP receptor components in the rat brain. Cephalalgia: an international journal of headache 39:342-353. Doi https://doi.org/10.1177/0333102417728873

52. Campos CA, Bowen AJ, Roman CW et al (2018) Encoding of danger by parabrachial CGRP neurons. Nature 555:617-622. Doi https://doi.org/10. 1038/nature25511

53. Han JS, Adwanikar H, Li Z et al (2010) Facilitation of synaptic transmission and pain responses by CGRP in the amygdala of normal rats. Mol Pain 6:10. Doi https://doi.org/10.1186/1744-8069-6-10

54. Ehrlich I, Klein M, Rumpel S et al (2007) PSD-95 is required for activitydriven synapse stabilization. P Natl Acad Sci Usa 104:4176-4181. Doi https://doi.org/10.1073/pnas.0609307104

55. Thiel G (1993) Synapsin I, synapsin II, and synaptophysin: marker proteins of synaptic vesicles. Brain Pathol 3:87-95. Doi https://doi.org/10.1111/j. 1750-3639.1993.tb00729.x

56. Valtorta F, Pennuto M, Bonanomi D et al (2004) Synaptophysin: leading actor or walk-on role in synaptic vesicle exocytosis? BioEssays: news and reviews in molecular, cellular and developmental biology. 26:445-453. https://doi.org/10.1002/bies.20012
57. Bradberry MM, Courtney NA, Dominguez MJ et al (2020) Molecular Basis for Synaptotagmin-1-Associated Neurodevelopmental Disorder. Neuron 107:52-64. Doi https://doi.org/10.1016/j.neuron.2020.04.003

58. Park Y, Ryu JK (2018) Models of synaptotagmin-1 to trigger Ca -dependent vesicle fusion. Febs Lett 592:3480-3492. Doi https://doi.org/10.1002/ 1873-3468.13193

59. Okutsu $Y$, Takahashi $Y$, Nagase $M$ et al (2017) Potentiation of NMDA receptor-mediated synaptic transmission at the parabrachial-central amygdala synapses by CGRP in mice. Mol Pain 13:2071454319. Doi https://doi.org/ 10.1177/1744806917709201

60. Dickstein DL, Weaver CM, Luebke Jl et al (2013) Dendritic spine changes associated with normal aging. Neuroscience 251:21-32. Doi https://doi. org/10.1016/j.neuroscience.2012.09.077

61. Hongpaisan J, Xu C, Sen A et al (2013) PKC activation during training restores mushroom spine synapses and memory in the aged rat. Neurobiol Dis 55:44-62. Doi https://doi.org/10.1016/j.nbd.2013.03.012

62. Sarajärvi T, Jäntti M, Paldanius K et al (2018) Protein kinase C -activating isophthalate derivatives mitigate Alzheimer's disease-related cellular alterations. Neuropharmacology 141:76-88. Doi https://doi.org/10.1016/j. neuropharm.2018.08.020

63. Sun MK, Alkon DL (2014) The "memory kinases": roles of PKC isoforms in signal processing and memory formation. Prog Mol Biol Transl 122:31-59. Doi 10.1016/B978-0-12-420170-5.00002-7

64. Zhang H, Macara IG (2008) The PAR-6 polarity protein regulates dendritic spine morphogenesis through p190 RhoGAP and the Rho GTPase. Dev Cell 14:216-226. Doi https://doi.org/10.1016/j.devcel.2007.11.020

\section{Publisher's Note}

Springer Nature remains neutral with regard to jurisdictional claims in published maps and institutional affiliations.

Ready to submit your research? Choose BMC and benefit from:

- fast, convenient online submission

- thorough peer review by experienced researchers in your field

- rapid publication on acceptance

- support for research data, including large and complex data types

- gold Open Access which fosters wider collaboration and increased citations

- maximum visibility for your research: over 100M website views per year

At BMC, research is always in progress.

Learn more biomedcentral.com/submissions 